\title{
July 2013 Pulmonary Case of the Month: Swan Song
}

\author{
Bridgett Ronan, MD \\ Lewis J. Wesselius, MD \\ Department of Pulmonary Medicine \\ Mayo Clinic Arizona \\ Scottsdale, AZ
}

\section{History of Present IIIness}

A 53 year old man presented to the emergency department with a 2 week history of progressive dyspnea. He thought it was anxiety due to quitting drinking just before the onset of his symptoms. He also had fatigue and malaise.

\section{$\mathrm{PMH}, \mathrm{SH}, \mathrm{FH}$}

He had no significant past medical history or family history. He did not smoke but drank 2-6 beers/day until 2 weeks prior to presentation.

\section{Physical Examination}

BP 110/60 mm Hg, P 110 beats/min, R 32 breaths $/ \mathrm{min}, \mathrm{T} 37.6^{\circ} \mathrm{C}, \mathrm{SpO} 281 \%$ $\mathrm{He}$ is pale and appears mildly dyspneic otherwise his physical exam is unremarkable.

\section{Chest Radiography}

His chest x-ray is shown in figure 1.

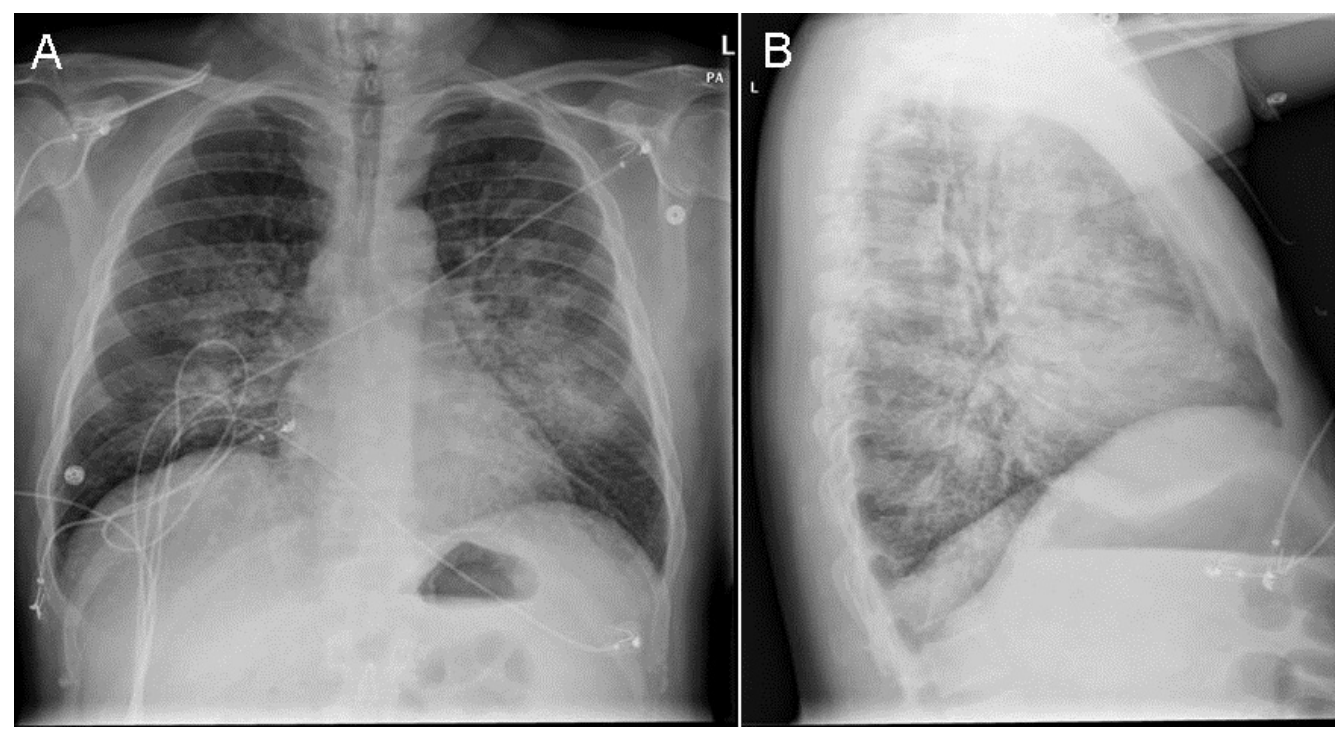

Figure 1. Initial PA (Panel A) and lateral (Panel B) chest X-ray. 
Which of the following laboratory tests is/are not indicated?

1. Arterial blood gases

2. Complete blood count

3. Spiral thoracic CT angiography

4. Urinanalysis

5. All of the above 


\section{Correct! \\ 3. Spiral thoracic CT angiography}

The patient's chest $x$-ray shows bilateral areas of lung consolidation and he is hypoxic (SpO2 81\%). At this juncture the etiology is unclear. Both an urinanalysis and complete blood count are indicated in the presence of diffuse lung consolidation and hypoxia since many lung diseases are also associated with abnormalities in the blood count and urinanalysis. A spiral thoracic CT angiogram is usually done for pulmonary embolism. However, the pre-test probability of pulmonary embolism is low in this case and a spiral CT angiogram should not be performed (1). He has had a 2 week long history of progressive dyspnea and has not known risk factors for pulmonary embolism.

His arterial blood gases showed a $\mathrm{PaO} 2$ of $46 \mathrm{~mm} \mathrm{Hg}, \mathrm{PaCO} 2$ of $28 \mathrm{~mm} \mathrm{Hg}, \mathrm{pH}$ of 7.53 and a HCO3- of $23 \mathrm{mEq} / \mathrm{L}$ on a non-rebreather mask. His hemoglobin is $6.0 \mathrm{~g} / \mathrm{dL}$, white blood cell count of 293,000 cells $/ \mathrm{mm} 3$ and a normal urinanalysis other than a specific gravity of 1.030 .

Which of the following is the best next step in the management of this patient?

1. Begin antibiotics for pneumonia

2. Endotracheal intubation

3. Leukapheresis to lower his white blood cell count

4. Packed red blood cell transfusion

5. All of the above 


\section{Correct! \\ 2. Endotracheal intubation}

The patient is severely ill. He is hypoxic despite his tachypnea and high inspired concentration of oxygen. The remaining choices might become necessary but the immediate concern is to control his airway and increase his PaO2. Intubation was accomplished uneventfully although his blood pressure did transiently decrease responding to a crystalloid fluid bolus. His urine output remained low. His SpO2 improved to $98 \%$ on an inspired oxygen concentration (FiO2) of $100 \%$. A portable chest x-ray was performed for endotracheal tube placement (Figure 2).

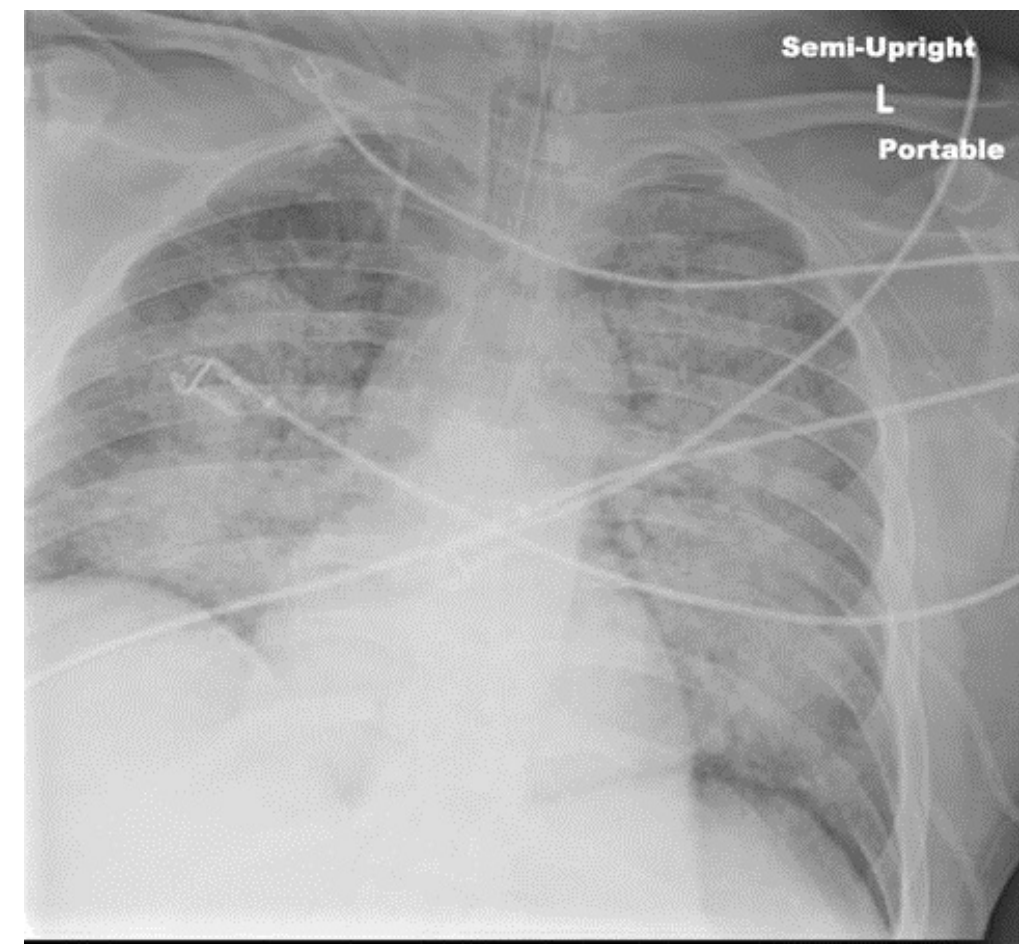

Figure 2. Portable AP of chest after endotracheal intubation.

A hematology consult is ordered and they diagnose acute leukemia.

Which of the following is the best explanation for his lung findings?

1. Alveolar hemorrhage

2. Granulomatosis with polyangiitis

3. Opportunistic infection

4. Pulmonary edema secondary to fluid administration

5. Pulmonary leukostasis 


\section{Correct! \\ 5. Pulmonary leukostasis}

Pulmonary edema secondary to fluid administration is unlikely because the patient was clinically dehydrated and at this juncture had received only 2 liters of fluid. An opportunistic infection is possible but he is afebrile and his history is more consistent with a slowly evolving process. There is nothing at this juncture to suggest alveolar hemorrhage or granulomatosis with polyangitis which is the new name for Wegener's granulomatosis. However, pulmonary leukostasis is likely in this situation $(2,3)$.

Pulmonary leukostasis can occur with hyperleukocytosis of any type. The critical leukocyte level for development of pulmonary leukostasis depends on the type of leukemia and the size of the cell. Generally, leukostasis does not occur until the white blood count excess 100,000 cells/mm3 for acute myelocytic leukemia, 150,000 cells $/ \mathrm{mm} 3$ for acute lymphocytic leukemia and 400,000 cells for chronic lymphocytic leukemia. Myelocytes and myeloblasts are the largest cell becoming progressively smaller with the lymphocytes in acute lymphocytic leukemia and even smaller with chronic lymphocytic leukemia. Multiple organs can be involved including the central nervous system and kidneys as well as the lungs.

There are 2 main theories of pulmonary leukostasis. One is that increased blood viscosity leads to sludging of blood in the pulmonary circulation. Leukemic blasts are less deformable than mature leukocytes. With high blast counts, plugs of cells develop in microcirculation, impeding blood flow. The other theory is that local hypoxemia and cytokine release result in pulmonary injury. The cells have high metabolic activity and release cytokines. The cytokines may lead to endothelial damage, resulting in hemorrhage and migration of leukemic cells into the tissue.

The patient was begun on cytoreduction therapy. Which of the following have not been proven to be beneficial in cytoreduction?

1. Chemotherapy

2. IV fluids

3. Leukapheresis

4. Pulmonary irradiation

5. Xanthine oxidase inhibitors 


\section{Correct! \\ 4. Pulmonary irradiation}

The goal in this patient was to reduce his white blood cell count. In general, pulmonary leukostasis improves with a reduction of the white count $(2,3)$. Leukapheresis will rapidly move white blood cells and was begun the night of admission. In addition, he was begun on chemotherapy consisting of daunorubicin, cytarabine, and hydroxyurea. Pulmonary irradiation is not helpful in resolving the pulmonary consolidation from pulmonary leukostasis (2). A major complication of treating very high white blood cell counts is tumor lysis syndrome (4). This occurs as a complication when large numbers of cancer cells die resulting in hyperkalemia, hyperphosphatemia, hyperuricemia and hyperuricosuria, hypocalcemia, and consequent acute uric acid nephropathy and acute renal failure. Large amounts of fluid were given along with xanthine oxidase inhibitors as prophylaxis against uric acid nephropathy. In addition he was treated empirically with broad-spectrum antibiotics as well as anti-fungals and anti-virals.

His white blood cell count decreased to 30,000 cells/mm3 and he remained relatively stable and afebrile. His platelet count was low but he was given intermittent platelet transfusions and vitamin $\mathrm{K}$ for intermittent mild hemoptysis. Although his FiO2 was high 0.60 , he demanded to be extubated on hospital day 3 . After extubation he was given oxygen by high flow nasal cannula. His arterial blood gases were $\mathrm{PaO} 2$ of $72 \mathrm{~mm} \mathrm{Hg}, \mathrm{PaCO} 2$ of $32 \mathrm{~mm} \mathrm{Hg}, \mathrm{pH}$ of 7.48 , and a HCO3- of $24 \mathrm{mEq} / \mathrm{L}$. A repeat portable chest x-ray was performed (Figure 3).

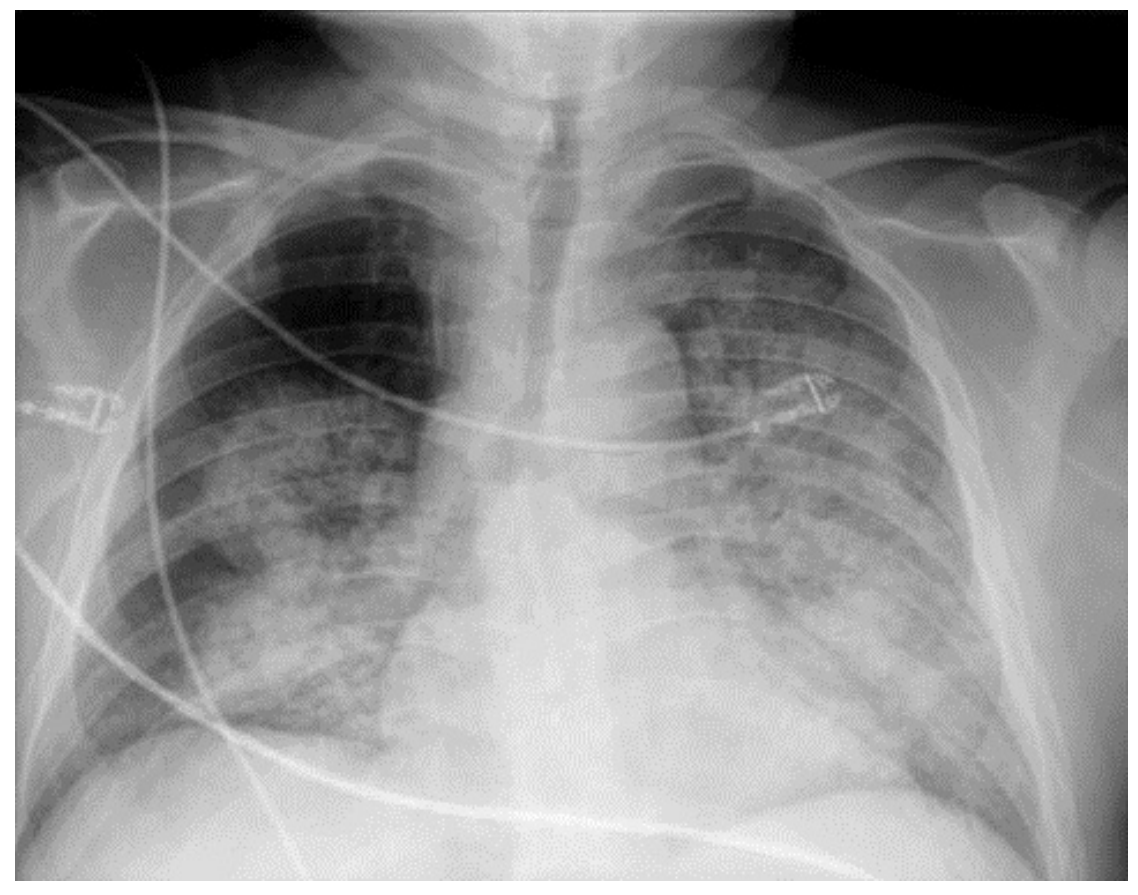

Figure 3. Portable AP of chest after extubation. 
A thoracic CT scan was performed because of his continuing hypoxia (Figure 4).

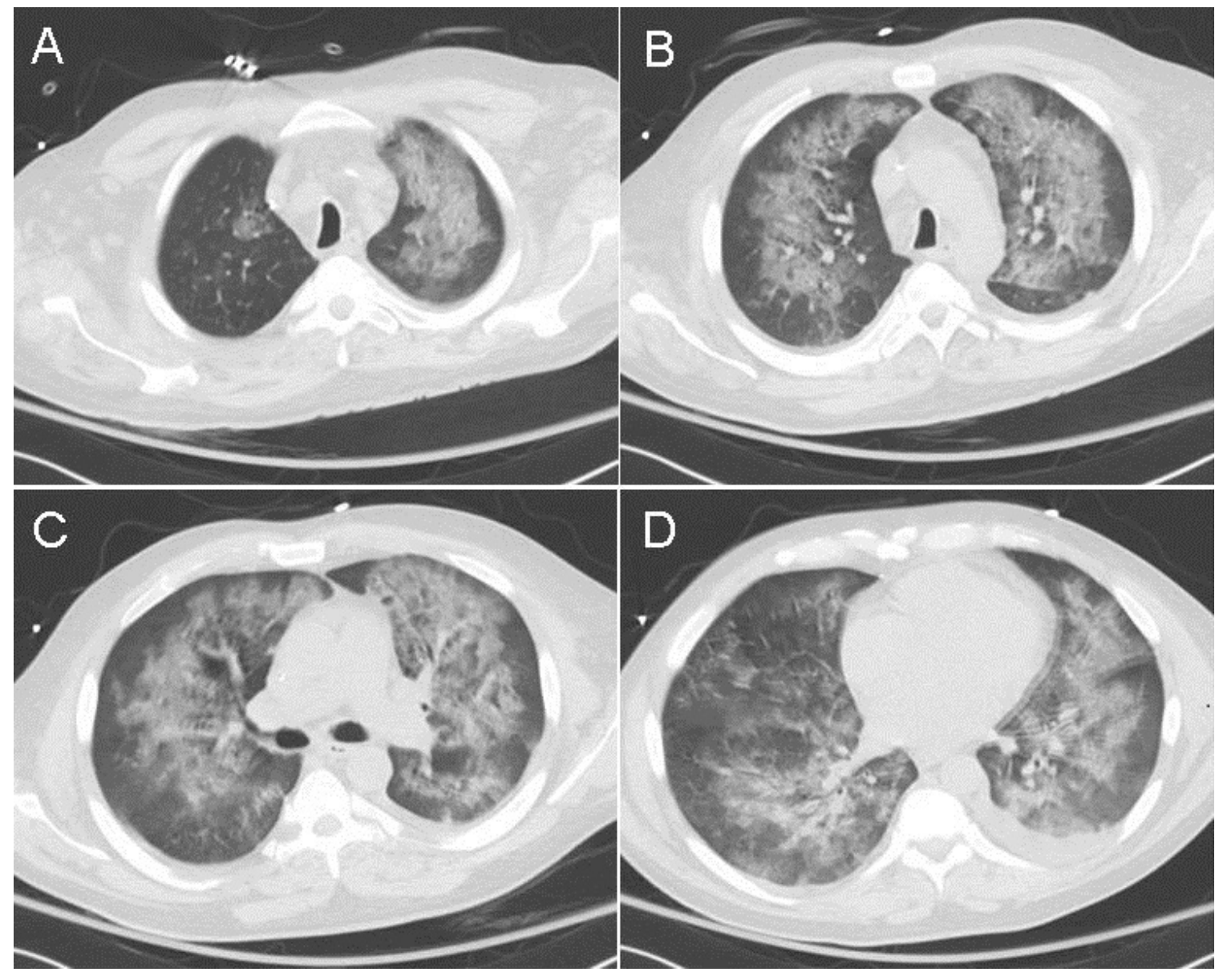

Figure 4. Representative static views from the thoracic CT scan.

Which of the following is the best explanation for his failure to resolve his ongoing pulmonary process?

1. Acute lysis pneumopathy

2. Alveolar hemorrhage

3. Drug-induced pulmonary injury

4. Opportunistic infection

5. Pulmonary edema 


\section{Correct! \\ 1. Acute lysis pneumopathy}

Any of the diagnosis is possible. However, acute lysis pneumopathy is the best choice $(5,6)$. This occurs when lysis of leukemic cells in the lung tissue release potentially harmful enzymes and possible other substances leading to diffuse alveolar damage (ARDS). Acute lysis pneumopathy usually occurs simultaneously with tumor lysis syndrome. The frequency of occurrence is unclear with it being described to occur "occasionally" up to $100 \%$ patients in one series (6).

It has been postulated that steroids may prevent acute lysis pneumopathy (6). Twenty patients with this disorder were given 10mg dexamethasone every 6 hours, and compared to historical controls had lower ICU mortality rate ( $20 \%$ vs. $50 \%$ ), less respiratory deterioration after chemotherapy (50\% vs. $80 \%)$ and no significant increases in infection.

Our patient was begun on dexamethasone, diuresed and continued on antibiotics. He clinically improved with his oxygen requirements decreasing to 2 $\mathrm{L} / \mathrm{min}$ by nasal cannula and his chest $\mathrm{x}$-ray improved (Figure 5).

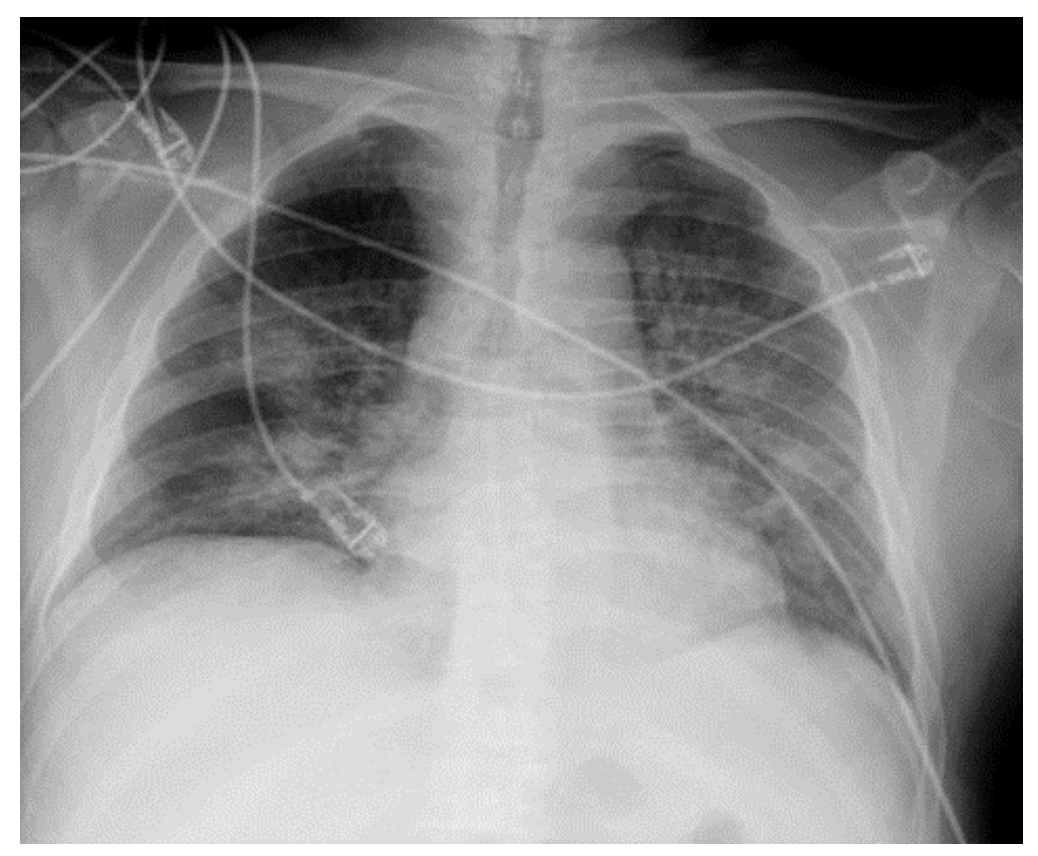

Figure 5. Portable AP of chest 2 days after beginning dexamethasone and diuresis.

Bridgett Ronan has contributed several cases of our Case of the Month series. She is completing her fellowship and this case is her "Swan Song" as a fellow. 


\section{References}

1. Stein PD, Woodard PK, Weg JG, Wakefield TW, Tapson VF, Sostman HD, Sos TA, Quinn DA, Leeper KV, Hull RD, Hales CA, Gottschalk A, Goodman LR, Fowler SE, Buckley JD. Diagnostic pathways in acute pulmonary embolism: recommendations of the PIOPED II Investigators. Radiology 2007;242(1):15-21. [CrossRef] [PubMed]

2. Ganzel C, Becker J, Mintz PD, Lazarus HM, Rowe JM. Hyperleukocytosis, leukostasis and leukapheresis: practice management. Blood Rev. 2012;26(3):117-22. [CrossRef] [PubMed]

3. Piro E, Carillio G, Levato L, Kropp M, Molica S. Reversal of leukostasisrelated pulmonary distress syndrome after leukapheresis and low-dose chemotherapy in acute myeloid leukemia. J Clin Oncol. 2011;29(26):e725-6. [CrossRef] [PubMed]

4. Howard SC, Jones DP, Pui $\mathrm{CH}$. The tumor lysis syndrome. N Engl J Med. 2011;364(19):1844-54. [CrossRef] [PubMed]

5. Tryka AF, Godleski JJ, Fanta CH. Leukemic cell lysis pneumonopathy. A complication of treated myeloblastic leukemia. Cancer. 1982;50(12):2763-70. [CrossRef] [PubMed]

6. Azoulay É, Canet E, Raffoux E, Lengliné E, Lemiale V, Vincent $F$, de Labarthe A, Seguin A, Boissel N, Dombret H, Schlemmer B. Dexamethasone in patients with acute lung injury from acute monocytic leukaemia. Eur Respir J. 2012;39(3):648-53. [CrossRef] [PubMed] 